\section{(6) OPEN ACCESS}

\title{
Self-management approaches for osteoarthritis in the hand: a $2 \times 2$ factorial randomised trial
}

\author{
Krysia Dziedzic, ${ }^{1}$ Elaine Nicholls, ${ }^{1}$ Susan Hill, ${ }^{1}$ Alison Hammond, ${ }^{2}$ June Handy, ${ }^{1}$ \\ Elaine Thomas, ${ }^{1}$ Elaine Hay ${ }^{1}$
}

\begin{abstract}
Handling editor Tore K Kvien
- Additional material is published online only. To view please visit the journal online (http://dx.doi.org/10.1136/ annrheumdis-2013-203938).

${ }^{1}$ Arthritis Research UK Primary Care Centre, Keele University, Keele, UK

${ }^{2}$ Centre for Health Sciences Research, University of Salford Salford, UK
\end{abstract}

\section{Correspondence to} Professor Krysia Dziedzic, Arthritis Research UK Primary Care Centre, Keele, Staffordshire ST5 5BG, UK; k.s.dziedzic@keele.ac.uk

Received 13 May 2013 Revised 2 August 2013 Accepted 17 September 2013 Published Online First 9 October 2013
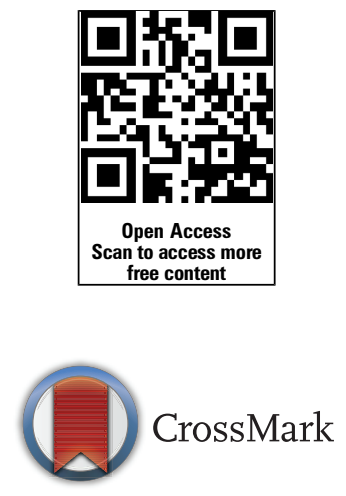

\footnotetext{
To cite: Dziedzic $K_{\text {, }}$ Nicholls E, Hill S, et al. Ann Rheum Dis 2015;74: 108-118.
}

\section{ABSTRACT}

Background Osteoarthritis is the leading cause of disability in older adults. Evidence of effectiveness for self-management of hand osteoarthritis is lacking. Methods In this randomised, factorial trial, we evaluated the effectiveness of joint protection versus no joint protection, and hand exercise versus no hand exercise in adults, 50 years of age or older, with hand osteoarthritis. Following a population survey $(n=12297)$, eligible individuals were randomly assigned (1:1:1:1) to: leaflet and advice; joint protection; hand exercise; joint protection plus hand exercise. Joint protection and hand exercises were delivered by nine occupational therapists, over four group sessions. The primary outcome was the OARSI/OMERACT responder criteria at 6 months. Outcomes were collected blind to allocation $(3,6$, $12 \mathrm{~m})$. Analysis was by intention to treat.

Results of 257 participants randomised (65:62:65:65) (mean age (SD) 66 years (9.1); female 66\%) follow-up was $85 \%$ at $6 \mathrm{~m}(n=212)$. Baseline characteristics and loss to follow-up were similar between groups. There were no reported treatment side effects. At $6 \mathrm{~m} \mathrm{33 \%}$ assigned joint protection were responders compared with $21 \%$ with no joint protection $(p=0.03)$. Of those assigned hand exercises, 28\% were responders compared with $25 \%$ with no exercises (n.s.). Differences in secondary outcomes were not statistically significant, except for improvement in pain self-efficacy with joint protection ( $3 \mathrm{~m} \mathrm{p}=0.002 ; 6 \mathrm{~m} \mathrm{p}=0.001 ; 12 \mathrm{~m} \mathrm{p}=0.03$ ). Conclusions These findings show that occupational therapists can support self-management in older adults with hand osteoarthritis, and that joint protection provides an effective intervention for medium term outcome. (Funded by the Arthritis Research UK ISRCTN 33870549).

\section{INTRODUCTION}

Osteoarthritis (OA) is the commonest form of arthritis in the Western world, and is the leading cause of disability in older adults, with the hand being one of the most frequently affected sites. ${ }^{1}$ In a country the size of the USA, conservative estimates suggest that there are 12.4 million people aged 65 years and over, with OA $(33.6 \%),{ }^{2}$ and 2.9 million adults aged 60 years and over with painful, disabling hand $\mathrm{OA}^{3}$ ${ }^{4}$ which significantly restricts daily activities, such as dressing and bathing, and evidence shows patients and practitioners perceive that there is little that can be done..$^{6}$ The majority of people with hand OA are managed in primary care but often treatments recommended by guidelines, for example, European
League Against Rheumatism (EULAR) recommendations, are not offered. ${ }^{7}$

Community-based self-management programmes have been proposed generally by national bodies in the USA and Europe as a potential, cost-effective approach for reducing the impact of OA. ${ }^{8}{ }^{9}$ While a number of authors have reviewed the effectiveness of self-management programmes for people with $\mathrm{OA},{ }^{7} 10$ or evaluated hand $\mathrm{OA}$ programmes in secondary care, ${ }^{11} 12$ as yet there is limited evidence for effective management of hand $\mathrm{OA}$ in communitydwelling populations.

Here we report the findings from the first large-scale randomised trial to investigate the clinical effectiveness of two self-management programmes for community-dwelling adults aged 50 years and over with hand OA. This multicentre two-by-two factorial randomised controlled trial addresses the following questions: Is joint protection education delivered by an occupational therapist (OT) more effective in reducing hand pain and disability than no joint protection education in people with hand OA? Is instruction in hand exercises delivered by an OT more effective in reducing hand pain and disability than no instruction in hand exercises in people with hand OA?

\section{METHODS}

\section{Design overview}

This was a randomised controlled factorial trial. The main comparisons were between joint protection and no joint protection, and between hand exercises and no hand exercises.

The Self Management in OA of the Hand (SMOotH) trial was conducted from June 2008 through May 2010 at the Arthritis Research UK Primary Care Centre, Keele University, UK. The trial was approved by the North West 7 Research Ethics Committee UK (rec reference: 07/H1008/ 235) and was monitored by an Independent Trial Steering Committee and Data Monitoring Committee (Trial registration number ISRCTN 33870549). The protocol, including the statistical analysis plan, has been published previously. ${ }^{13}$

\section{Setting and participants}

Participants aged 50 years and over, registered with five general practices in Central Cheshire and North Staffordshire, UK, were mailed a health survey between June 2008 and April 2009. Responders to the health survey were invited for an assessment at a research clinic to check eligibility for the trial if they: (1) gave consent to further 
contact; (2) reported hand pain in the last 12 months; (3) reported hand pain, aching or stiffness on 'some days', 'most days' or 'all days' in the last month; (4) had an Australian Canadian Hand OA Outcomes Index (AUSCAN) pain score $\geq 5$ or an AUSCAN function score $\geq 9^{14}{ }^{15}$; (5) reported that they had not seen an OT or physiotherapist for their hand problem in the last 6 months; (6) had not had a hand operation, injection nor injured their hands badly enough to see a doctor in the previous 6 months and (7) had no other member of their household participating in the trial. These individuals were mailed a study information sheet about the trial, and a letter inviting them to telephone the research centre should they wish to attend the research clinic.

Those attending the research clinic were assessed for trial eligibility by a research nurse and were included in the trial if they: gave informed consent to participate in the trial; met the American College of Rheumatology (ACR) criteria for features of hand $\mathrm{OA},{ }^{16}{ }^{17}$ or had unilateral or bilateral thumb base OA; did not have an alternative clinical diagnosis, such as inflammatory arthritis, and were able to attend for the trial interventions at participating OT departments. Details of consenting eligible participants were forwarded to the research centre and participants were randomised via a remote randomisation service to one of four groups (see below).

\section{Randomisation and interventions}

Randomisation was conducted (with an allocation ratio of $1: 1: 1: 1)$ by administrative staff at the Keele Musculoskeletal Clinical Trials Unit, Arthritis Research UK Primary Care Centre, who had no clinical involvement in the trial. Randomisation was stratified by participants' general practice, and was completed using random permuted blocks of size 4 (the blocks were randomly selected using a computer-generated random number sequence in an ACCESS database). Randomised allocation of the next patient was concealed from administrative and OT staff until the point of randomisation. Researchers who entered and analysed the data were unaware of treatment allocation.

Participants were randomised to one of four 'cells': (1) joint protection; (2) hand exercises; (3) joint protection and hand exercises combined; (4) no joint protection or hand exercises. Participants in this last cell received written advice only and did not receive occupational therapy sessions. Interventions delivered in all four cells are described in Box 1.

Twelve OTs attended a two-day training programme before delivering the joint protection education and instruction in hand exercises (see online supplementary text S1). A pilot study tested the intervention protocol prior to the commencement of the main trial.

\section{Outcomes and follow-up}

Study outcomes were collected at baseline, 3, 6 and 12 months post-randomisation by postal questionnaire, and in a clinical assessment at 6 months. The primary end point for the trial was at 6-month follow-up. The study was designed so that the research nurse conducting the 6-month clinical assessment was blind to treatment allocation. However, if un-blinding did occur this was recorded.

The primary outcome measure combines the pain and function subscales of the AUSCAN ${ }^{14}{ }^{15}$ and global assessment of change ${ }^{23}$ to determine if participants were 'responders' to treatment using the OARSI-OMERACT criteria ${ }^{24}$ (for further details see footnote to table 1).

Self-reported secondary outcomes included AUSCAN pain, stiffness and function, global assessment of change in hand problem, average pain severity over the past 3 days (0-10 numerical rating scale), severity rating of participant-nominated main functional problem over the past 3 days (0-10 numerical rating scale), satisfaction with hand function over the past 3 days (0-10 numerical rating scale), health-related quality of life as measured by the SF12v2, ${ }^{25}$ and the Arthritis Self Efficacy pain subscale. ${ }^{26}$ Additionally, the following secondary outcomes were measured by a research nurse at baseline and at the 6 -month clinical assessment only ${ }^{13}$ : (1) grip strength (JAMAR); (2) pinch strength (B \& L pinch gauge); (3) functional performance using the grip ability test (GAT). ${ }^{27}$

\section{Treatment fidelity}

Self-reported performance of hand exercises and use of joint protection and energy conservation were recorded to assess level of adherence to the intervention. ${ }^{13} 22$

\section{Sample size}

In this factorial trial, the sample size calculation was based on the comparison of participants receiving hand exercises (intervention group) and not receiving hand exercises (comparator group), (the calculation would be identical for the comparison of joint protection vs no joint protection, as hand exercises and joint protection were assumed to be independent treatments). ${ }^{28}$ In participants in the comparator group, 50\% would receive only a leaflet and advice, and 50\% would receive joint protection education. Based on findings from similar populations of older adults with knee osteoarthritis, we estimated that $25 \%$ of participants receiving leaflet and advice only would improve using the OARSI-OMERACT responder criteria, and $45 \%$ of those receiving joint protection education would improve. ${ }^{24} 29$ This gave a combined improvement of $35 \%$ in participants in the comparator group, that is, those not receiving hand exercises, assuming equal allocation of participants between treatment groups.

Published information was not available to define a minimum clinical important difference for the primary outcome measure. Therefore, after a consensus discussion with the OTs delivering the trial interventions, we estimated a worthwhile difference between groups to be $20 \%$. Hence, the estimate of improvement in the intervention group which received hand exercises was 55\% (ie, between group differences $35 \%+20 \%$ ). To detect a difference of $20 \%$ or larger between participants receiving and those not receiving hand exercises, with $80 \%$ power and $\alpha$ of $5 \%$, a total of 212 participants with data at baseline and at 6 months was required. To allow for a $15 \%$ drop-out over the 6-month post-randomisation period, we planned to recruit 252 participants to the trial, that is, 126 per group for each comparison.

\section{Statistical analysis}

The main effectiveness analysis was completed on an intention to treat (ITT) basis with imputation of missing data (see online supplementary text S2 and S3 for full details of analysis methods). Continuous outcome measures were analysed using analysis of covariance (ANCOVA), and binary outcomes by logistic regression with treatment differences expressed as mean differences or ORs, as appropriate, with associated 95\% CIs. Descriptive statistics were used to describe baseline characteristics of participants by randomised treatment arm and by loss to follow-up. Adherence to hand exercises and use of joint protection techniques were analysed by treatment arm at 3, 6 and 12 months.

Sensitivity analyses of the trial results were conducted using (1) complete-case data and (2) per protocol data at 6 months using the primary outcome. Analyses were completed in STATA 


\section{Box 1 Interventions delivered to participants}

\section{Leaflet and advice}

All participants were given standardised written information on self-management approaches for hand osteoarthritis (OA) including general information on looking after hand joints, and using analgesia (reproduced with permission from the Arthritis Research UK leaflets 'Looking after your joints when you have arthritis' and 'Osteoarthritis', respectively (http://www.arthritisresearchuk.org/), and the National Institute of Health and Care Excellence (NICE) good practice guidelines. ${ }^{18}$ Participants were advised to continue with any self-management approaches they were currently using, and were given advice to consult their general practitioner if symptoms continued to be troublesome.

For $25 \%$ of participants this was the sole intervention.

\section{Joint protection, hand exercises}

For the remaining $75 \%$ of participants, in addition to receiving the leaflet, they received one of three interventions: joint protection, hand exercises, or a combination of the two. The interventions were all delivered over four group sessions (held once a week) by nine occupational therapists (OTs) in two hospital centres. OTs were rotated every 3 months to minimise the potential for bias. The rotation order was determined by the OTs availability to deliver the specific intervention.

Groups included up to six participants, and lasted for a maximum of one hour (1.5 h for the combined intervention). Treatment session duration and participant attendances were recorded by the OTs on case report forms (CRFs). Attendance adherence was audited by the study coordinator (SH), and was defined (a priori) to be per protocol if participants attended: session 1, 2, 3 and 4; sessions 1, 2 and 4; sessions 1, 3 and 4; or sessions 1 and 4. Any participant unable to attend week 1 was booked on to the following course.

\section{Joint protection, hand exercises: core components}

Both interventions were based on the 'Looking After Your Joints Programme' for rheumatoid arthritis (RA), ${ }^{19-22}$ and included the following core components:

- a general introduction to the programme

- education about hand $\mathrm{OA}$ and its management

- managing pain during everyday activities

- how to change habits

- long-term and short-term goal setting

- weekly individually negotiated home programmes to practise skills taught

- weekly review of individually negotiated home programmes

Participants were provided with workbooks (relevant to joint protection, hand exercises, or a combination of the two) including key points from each meeting, photographs of people with hand OA demonstrating the intervention and weekly activity diaries to complete.

\section{Joint protection principles}

In addition to the core components outlined above, this intervention included the following joint protection principles:

- distributing the weight of what you lift over several joints (eg, spread the load over two hands)

- avoiding putting strain on the thumb and repetitive thumb movements

- avoiding prolonged grips in one position

- using as large a grip as possible

- reducing the effort needed to do a task (eg, use labour-saving gadgets; avoid lifting heavy objects, and reduce the weight of what you lift)

- energy conservation (activity pacing and planning)

\section{Hand exercises}

In addition to the core components outlined above, this intervention included the following stretching and strengthening hand and thumb exercises:

- stretching exercises

- wrist flexion and extension, pronation and supination

- tendon gliding

- radial finger walking

- making an ' 0 ' with the thumb and index finger

- thumb extension, abduction and opposition to the base of the 5 th finger

\section{Strengthening exercises}

- using an elastic band to provide resistance to thumb extension, thumb abduction and finger extension

- using Play-Doh rolling and forming into a ring to provide resistance to thumb and finger extension, squeezing it into a ball, and pinching off pieces between the thumb and index fingers

- holding a $0.5-0.75 \mathrm{~kg}$ weight while doing wrist flexion and extension exercises in pronation then supination

The aims of the hand exercise programme were to give the participants a clearer understanding of their hand problem, and to develop a hand exercise routine to help them improve grip strength and dexterity. Participants were guided to start with three repetitions of each exercise, gradually building up to 10 repetitions of each exercise daily (or most days), and to perform the exercises within their limit of discomfort. Exercises could be spread over several exercise sessions during the day and performed more than once a day. Participants were also asked to write down how many times they aimed to practise the exercises.

\section{Joint protection and hand exercises combined}

The individual interventions as described above were combined in the same number of sessions but with an additional half an hour added to each session. 
Table 1 Baseline characteristics of randomised participants

\begin{tabular}{|c|c|c|c|c|c|}
\hline Characteristic & $\begin{array}{l}\text { Leaflet and advice } \\
\text { ( } \mathrm{L} \text { and } A) \\
n=65\end{array}$ & $\begin{array}{l}\text { Joint protection } \\
\text { (JP) } \\
n=62\end{array}$ & $\begin{array}{l}\text { Hand exercises } \\
\text { (HEx) } \\
n=65\end{array}$ & $\begin{array}{l}\text { Combined therapy } \\
\text { (JP and HEx) } \\
n=65\end{array}$ & $\begin{array}{l}\text { All randomised } \\
\mathrm{n}=257\end{array}$ \\
\hline \multicolumn{6}{|l|}{ Demographic data } \\
\hline Mean (SD): Age (years) & $67.2(9.5)$ & $65.5(8.6)$ & $64.5(9.0)$ & $66.0(9.3)$ & $65.8(9.1)$ \\
\hline Female & $40(62)$ & $43(69)$ & $41(63)$ & $46(71)$ & $170(66)$ \\
\hline Married & $44(69)$ & $43(71)$ & $36(55)$ & $42(65)$ & $165(65)$ \\
\hline Routine or manual occupation* $\dagger$ & $34(52)$ & $24(39)$ & $32(49)$ & $31(48)$ & $121(47)$ \\
\hline Currently working & $20(31)$ & $18(29)$ & $20(31)$ & $18(28)$ & $76(30)$ \\
\hline Mean (SD): Age when left school & $15(1.1)$ & $16(1.4)$ & $16(1.2)$ & $15(1.2)$ & $16(1.2)$ \\
\hline Left school to go to full-time education or university & $9(14)$ & $13(21)$ & $12(19)$ & $7(11)$ & $41(16)$ \\
\hline Gained qualifications through study as an adult & $25(40)$ & $36(60)$ & $36(55)$ & $29(45)$ & $126(50)$ \\
\hline \multicolumn{6}{|l|}{ General health and quality of life } \\
\hline Body Mass Index $\geq 25.0 \mathrm{~kg} / \mathrm{m}^{2}$ (overweight/obese) $\ddagger$ & $39(63)$ & $41(71)$ & $48(75)$ & $41(65)$ & $169(68)$ \\
\hline Mean (SD): SF-12: Physical component $(0-100)^{*}$ & $39.7(12.5)$ & $39.0(10.4)$ & $41.9(9.5)$ & $39.9(10.1)$ & $40.1(10.7)$ \\
\hline Median (IQR): SF-12: Mental component $(0-100)^{*}$ & $52.2(44.0,58.0)$ & $55.5(47.7,60.0)$ & $50.5(39.9,57.7)$ & $56.4(43.0,60.5)$ & $53.4(43.3,59.2)$ \\
\hline \multicolumn{6}{|l|}{ Clinical characteristics of hand problem } \\
\hline Pain in both hands in last 12 months & $60(92)$ & $52(84)$ & $57(88)$ & $56(88)$ & $225(88)$ \\
\hline Median (IQR): Number of years with hand problem* & $5.0(3.0,10.0)$ & $4.0(2.0,8.0)$ & $5.0(2.0,10.0)$ & $5.0(2.0,10.0)$ & $5.0(2.0,10.0)$ \\
\hline Mean (SD): AUSCAN-pain $(0-20)^{*}$ & $9.5(4.0)$ & $10.2(3.5)$ & $8.8(3.3)$ & $9.4(3.7)$ & $9.4(3.6)$ \\
\hline Mean (SD): AUSCAN—stiffness $(0-4)^{*}$ & $1.6(1.0)$ & $1.5(1.1)$ & $1.4(1.0)$ & $1.5(1.1)$ & $1.5(1.0)$ \\
\hline Mean (SD): AUSCAN-function $(0-36)^{*}$ & $14.5(8.0)$ & $15.9(7.9)$ & $13.8(7.2)$ & $15.0(7.3)$ & $14.8(7.6)$ \\
\hline Mean (SD): AUSCAN-total $(0-12)^{*}$ & $5.2(2.2)$ & $5.3(2.4)$ & $4.7(1.9)$ & $5.0(2.3)$ & $5.0(2.2)$ \\
\hline Mean (SD): Arthritis self-efficacy pain subscale $(1-10)^{*}$ & $4.8(1.9)$ & $5.3(1.7)$ & $5.2(1.8)$ & $5.1(1.6)$ & $5.1(1.8)$ \\
\hline $\begin{array}{l}\text { Mean (SD): Hand pain severity on average last } 3 \text { days } \\
(0-10)^{*}\end{array}$ & $4.7(2.2)$ & $5.2(2.1)$ & $4.3(1.8)$ & $4.4(1.9)$ & $4.6(2.0)$ \\
\hline $\begin{array}{l}\text { Mean (SD): Severity of main functional problem on } \\
\text { average in the last } 3 \text { days }(0-10)^{*}\end{array}$ & $4.9(2.3)$ & $5.6(2.5)$ & $4.7(2.4)$ & $5.0(2.3)$ & $5.0(2.4)$ \\
\hline $\begin{array}{l}\text { Mean (SD): Satisfaction with hand function last } 3 \text { days } \\
(0-10)^{*}\end{array}$ & $4.6(2.2)$ & $5.4(2.5)$ & $4.6(2.4)$ & $4.4(2.0)$ & $4.8(2.3)$ \\
\hline Median (IQR): Grip strength (Ibs)* & $35.0(25.5,47.5)$ & $31.8(17.0,47.5)$ & $31.0(21.5,51.0)$ & $33.5(22.5,48.5)$ & $33.5(22.5,47.5)$ \\
\hline Mean (SD): Pinch strength (lbs)* & $8.8(3.3)$ & $8.8(3.8)$ & $9.0(3.2)$ & $9.0(3.3)$ & $8.9(3.4)$ \\
\hline Median (IQR): Grip ability test (seconds)* & $32.2(26.8,43.6)$ & $30.2(24.9,39.7)$ & $32.2(26.6,35.8)$ & $32.1(27.2,41.5)$ & $32.0(26.5,40.4)$ \\
\hline ACR criteria met§ & $59(91)$ & $56(90)$ & $59(91)$ & $56(86)$ & $230(90)$ \\
\hline Unilateral or bilateral thumb OAף & $50(77)$ & $52(84)$ & $49(75)$ & $59(91)$ & $210(82)$ \\
\hline \multicolumn{6}{|c|}{$\begin{array}{l}\text { Figures are numbers and percentages unless otherwise stated. Median (IQR) given for outcome measures with a skewed distribution. Total AUSCAN score calculated as (pain/5) } \\
\text { +stiffness+(function/9). } \\
\text { `Data based on imputed data. } \\
\text { †Based on the 'lower supervisory/technical', 'Semiroutine' and 'Routine' groups of the UK Standard Occupation Classification (2000) for current or most recent paid employment. } \\
\text { ¥Body Mass Index grouping defined according to the WHO. } \\
\text { §ACR criteria based on clinical features only (symptom frequency assessed prior to clinical assessment). } \\
\text { IThe eligibility criteria for thumb base OA were determined via examination of the hand joints for features of pain and/or tenderness on palpation and observation/palpation of } \\
\text { deformity. This definition of thumb OA is in keeping with NICE recommendations for diagnosis of OA in UK Primary Care. }{ }^{18} \\
\text { ACR, American College of Rheumatology; AUSCAN, Australian/Canadian Hand Osteoarthritis Index; JP, joint protection; NICE, National Institute of Health and Care Excellence; OA, } \\
\text { osteoarthritis; SF-12, Short Form Health Survey } 12 \text { (V.2). }\end{array}$} \\
\hline
\end{tabular}

12.0. ${ }^{30}$ No interim analyses were undertaken during the trial or follow-up period.

\section{Role of the funding source}

The trial was funded by the Arthritis Research UK ISRCTN 33870549. The funder played no role in the study.

\section{RESULTS}

\section{Study recruitment and follow-up}

Trial eligibility, recruitment and follow-up are described in figure 1. Overall follow-up rates (including minimum data collection) were: 3 months, 90\% $(\mathrm{n}=232), 6$ months, $85 \%$ $(n=218), 12$ months, $85 \%(n=219)$. Rates of loss to follow-up were similar for each intervention arm (figure 1) and were not related to baseline participant characteristics (see online supplementary table S1).
Table 1 shows baseline characteristics of trial participants. The mean (SD) age of participants was 66 (9.1) years, 66\% were female; the mean (SD) AUSCAN pain and function scores at trial entry were: pain 9.4 (3.6); function 14.8 (7.6). Overall, differences in participant characteristics across treatment arms at baseline were small, however, some between-group differences were observed for gender, marital status, social class, Body Mass Index and the presence of thumb OA.

\section{Main trial results}

Interaction terms for the primary outcome (see online supplementary table S2) and all other outcomes were not statistically significant $(\mathrm{p} \geq 0.05)$, therefore, treatment effects were evaluated from the main effects model (ie, joint protection vs no joint protection; hand exercises vs no hand exercises) after adjustment for predefined potential confounders. 


\section{Clinical and epidemiological research}

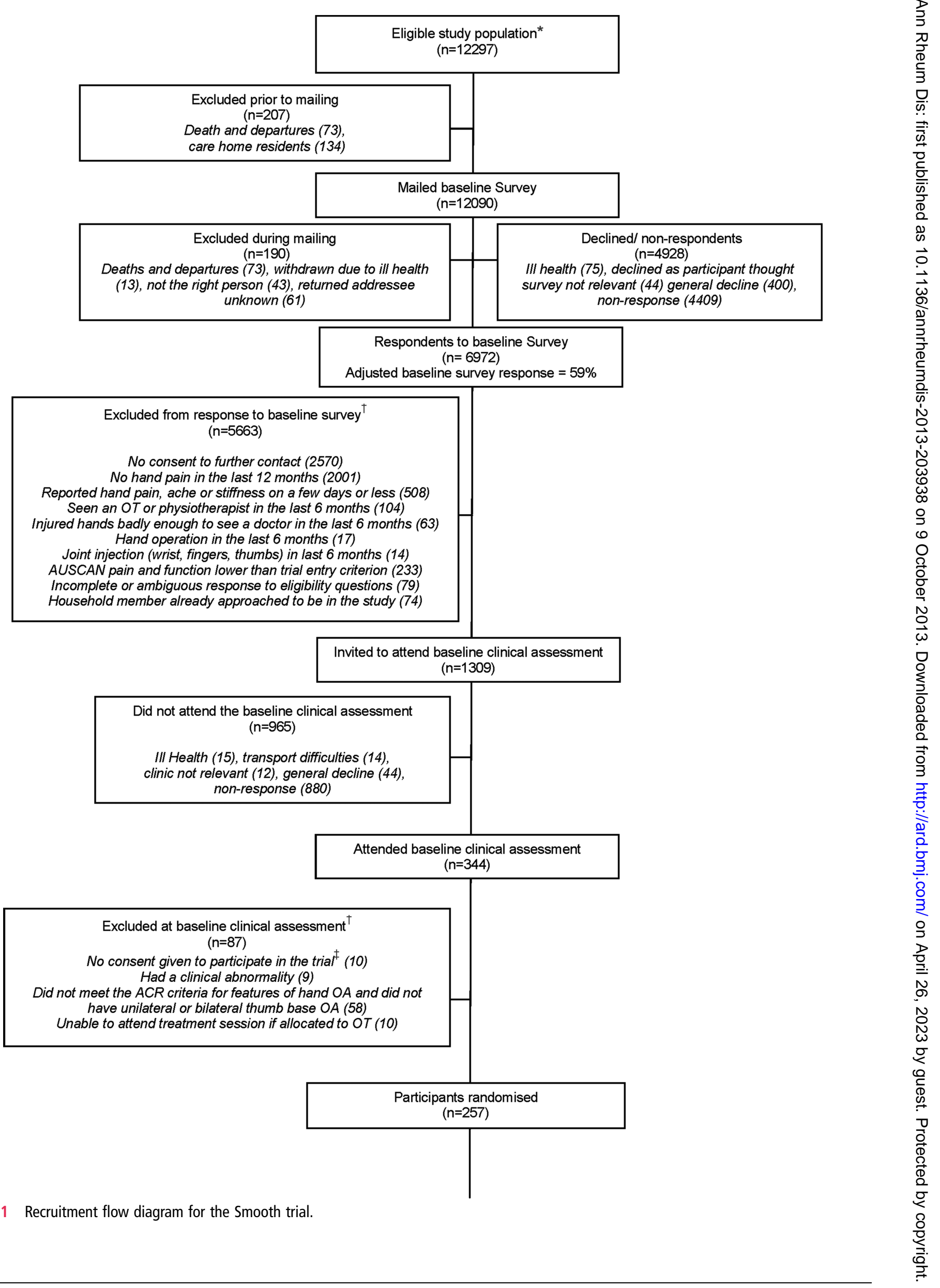




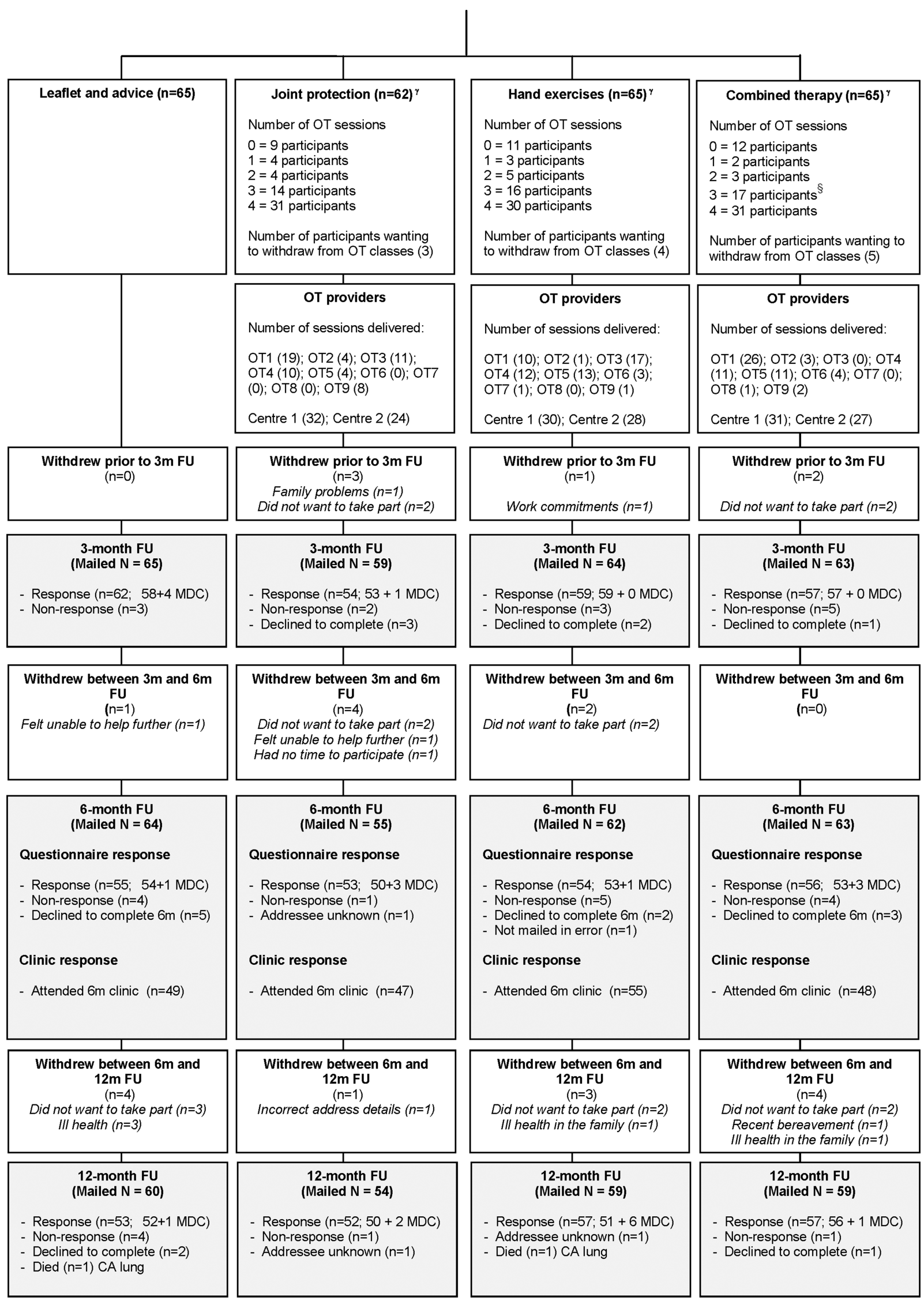

Figure 1 Continued. 


\section{Footnotes:}

* Eligible study population includes adults aged 50 years and over registered with one of the five study practices, who did not have any of the excluded conditions searched for at the GP practice, eg RA, and who were not excluded by the GP screen, eg vulnerable patients with a record of psychiatric or terminal illness. $\dagger$ Ineligibility reason is the first to apply on the list as multiple reasons are likely.

¥ This includes one red flag and one participant that withdrew consent after clinical attendance but prior to randomisation.

$\S$ A participant in this group attended four treatment sessions (session 1, 2, and 3, with session 3 repeated twice in error).

$\gamma$ Participants commenced occupational therapy in a median of 36 days (IQR 22,49) - joint protection 35

(IQR 22, 43); hand exercises 36 (IQR 22, 45); joint protection and hand exercises 44 (IQR 24, 64).

31 participants attended one or more OT class after the 3-month follow-up and 2 participants attended a single class after their 6-month follow-up.

Withdrawn indicates a request for no further study involvement; declined indicates a request that the participant does not want to provide data at the current follow-up but is willing to be mailed at further follow-ups. FU = follow-up; $\mathrm{MDC}=$ minimum data collection; 3m, 3-month, 6m, 6-month, 12m, 12-month.

Figure 1 Continued.

Table 2 shows the number (\%) of participants classified as 'responders' according to the OARSI criteria. At our primary end point (6 months), the proportion of people meeting the OARSI responder criteria was higher for joint protection (vs no joint protection) and hand exercises (vs no hand exercises). These differences reached statistical significance for the joint protection comparison. No statistically significant differences were observed at 3-month and 12-month follow-up for this measure.

Evaluation of the components of the OARSI responder criteria found no significant differences in mean AUSCAN pain or function subscales for either treatment comparison or any follow-up time point (table 3). The percentage of participants reporting global improvement was significantly higher in the groups receiving joint protection compared with no joint protection, and hand exercises versus no hand exercises at 6 months, and also at 3 months and 12 months for the hand exercises comparison only (table 3 and see online supplementary table S3).

From the remaining secondary outcomes (table 3), arthritis pain self-efficacy consistently showed statistically significant differences between those receiving, and those not receiving joint protection, at all time points, with participants receiving joint projection showing increased pain self-efficacy.

\section{Treatment fidelity}

Those allocated to hand exercises performed a structured exercise programme more often than those who were not (see online supplementary table S4). On average, participants allocated to joint protection used joint protection and energy conservation techniques more frequently than those who were not (see online supplementary table S4).

\section{Sensitivity analyses}

Overall, these findings were largely replicated in a complete case analysis with no imputation of missing data (see online supplementary table S5). A per-protocol analysis of the OARSI responder criteria at 6-month follow-up replicated results from the ITT analysis, although statistical significance of the joint protection comparison was marginal $(p=0.07)$ (see online supplementary table S6).

\section{Adverse events}

No adverse events were reported as a result of the interventions.

\section{DISCUSSION}

In this multicentre randomised controlled factorial trial, we evaluated whether joint protection education delivered by OTs was more effective in reducing hand pain and disability than no joint protection in community-dwelling older adults with hand OA. At 6 months, the primary end point, participants who received the joint protection intervention were statistically significantly more likely to be classified as responders to treatment than those not receiving joint protection (33\% cf 21\%). This was not maintained over 12 months. We also evaluated whether

Table 2 Treatment effectiveness evaluated for the OARSI responder criteria

\begin{tabular}{|c|c|c|c|c|c|c|c|c|c|c|c|c|}
\hline \multirow[b]{2}{*}{ Outcome measure } & \multicolumn{4}{|c|}{3 months } & \multicolumn{4}{|c|}{6 months } & \multicolumn{4}{|c|}{12 months } \\
\hline & No JP* & $J P$ & No HEx* & HEx & No JP* & $\mathrm{JP}$ & No HEx* & HEx & No JP* & JP & No HEx* & HEX \\
\hline$n$ & 130 & 127 & 127 & 130 & 130 & 127 & 127 & 130 & 130 & 127 & 127 & 130 \\
\hline 'Responders' (OARSI)†, n (\%) & $22(17)$ & $28(22)$ & $24(19)$ & $26(20)$ & $27(21)$ & $42(33)$ & $32(25)$ & $36(28)$ & $27(21)$ & $34(27)$ & $24(19)$ & $38(29)$ \\
\hline Adjusted $¥ \mathrm{OR}(95 \% \mathrm{Cl})$ & \multicolumn{2}{|c|}{1.35 (0.68 to 2.68$)$} & \multicolumn{2}{|c|}{$0.99(0.50$ to 1.95$)$} & \multicolumn{2}{|c|}{2.10 (1.09 to 4.04$)$} & \multicolumn{2}{|c|}{$1.14(0.59$ to 2.20$)$} & \multicolumn{2}{|c|}{$1.57(0.83$ to 3.00$)$} & \multicolumn{2}{|c|}{1.76 (0.93 to 3.34$)$} \\
\hline
\end{tabular}

* Reference category.
tParticipants met the OARSI responder criteria if (a) relative change in AUSCAN pain or function was $\geq 50 \%$ and absolute change was $\geq 20$ or (b) at least two of the following applied: relative change in pain $\geq 20 \%$ and absolute change $\geq 10$, relative change in function $\geq 20 \%$ and absolute change $\geq 10$ or participants reported they were better, much better, or completely recovered on the global assessment of change question. Absolute change (baseline-follow-up score) and relative change (absolute change/baseline score) were calculated after AUSCAN measures were scaled from 1 to 101 to avoid dividing by 0 when calculating relative change. ${ }^{24}$ Responses in the 'better', 'much better' or 'completely recovered' categories on the global assessment of change question were defined as 'improvement'.

¥Adjusted for age, gender, social class, General Practitioner (GP) practice and length of time with a hand condition and the other main effect of interest.

AUSCAN, Australian Canadian Hand Osteoarthritis Index; HEx, hand exercise; JP, joint protection; OA, Osteoarthritis. 
Table 3 Treatment effectiveness for secondary outcome measures by main treatment effects

\begin{tabular}{|c|c|c|c|c|c|c|c|c|c|c|c|c|}
\hline \multirow[b]{2}{*}{ Outcome measure } & \multicolumn{4}{|l|}{3 months } & \multicolumn{4}{|l|}{6 months } & \multicolumn{4}{|c|}{12 months } \\
\hline & No JP* & JP & No HEx* & HEx & No JP* & $J P$ & No HEx* & HEx & No JP* & $J P$ & No HEx* & HEx \\
\hline $\mathrm{n}$ & 130 & 127 & 127 & 130 & 130 & 127 & 127 & 130 & 130 & 127 & 127 & 130 \\
\hline \multicolumn{13}{|l|}{ Global assessment of change (\%) } \\
\hline $\mathrm{n}(\%)$ Improvedt & $39(30)$ & $43(34)$ & $29(23)$ & $53(41)$ & $30(23)$ & $52(41)$ & $28(22)$ & $55(42)$ & $34(26)$ & $46(36)$ & $28(22)$ & $51(39)$ \\
\hline Adjusted $\mathrm{OR}(95 \% \mathrm{Cl})$ & \multicolumn{2}{|c|}{$1.23(0.67$ to 2.25$)$} & \multicolumn{2}{|c|}{2.48 (1.33 to 4.60$)$} & \multicolumn{2}{|c|}{$2.71(1.39$ to 5.25$)$} & \multicolumn{2}{|c|}{2.79 (1.44 to 5.40$)$} & \multicolumn{2}{|c|}{$1.82(0.96$ to 3.45$)$} & \multicolumn{2}{|c|}{$2.22(1.20$ to 4.11$)$} \\
\hline \multicolumn{13}{|l|}{ AUSCAN pain $(0-20)$} \\
\hline Mean (SD) & $9.0(3.5)$ & $9.3(3.0)$ & $9.5(3.4)$ & $8.8(3.1)$ & $9.4(4.0)$ & $9.0(3.9)$ & $9.4(4.0)$ & $9.0(3.9)$ & $9.4(3.9)$ & $9.6(3.8)$ & $9.9(3.7)$ & $9.1(3.9)$ \\
\hline Adjusted mean difference $(95 \% \mathrm{Cl})$ & \multicolumn{2}{|c|}{$0.01(-0.71$ to 0.74$)$} & \multicolumn{2}{|c|}{$-0.31(-1.03$ to 0.42$)$} & \multicolumn{2}{|c|}{$-0.79(-1.70$ to 0.12$)$} & \multicolumn{2}{|c|}{$0.06(-0.85$ to 0.97$)$} & \multicolumn{2}{|c|}{-0.09 ( -0.99 to 0.81$)$} & \multicolumn{2}{|c|}{$-0.35(-1.27$ to 0.56$)$} \\
\hline \multicolumn{13}{|l|}{ AUSCAN stiffness $(0-4)$} \\
\hline Mean (SD) & $1.5(1.0)$ & $1.5(1.0)$ & $1.6(1.0)$ & $1.4(1.0)$ & $1.6(1.0)$ & $1.4(1.0)$ & $1.5(1.0)$ & $1.5(1.0)$ & $1.6(1.0)$ & $1.5(1.0)$ & $1.7(0.9)$ & $1.5(1.0)$ \\
\hline Adjusted mean difference $(95 \% \mathrm{Cl})$ & \multicolumn{2}{|c|}{$0.02(-0.19$ to 0.22$)$} & \multicolumn{2}{|c|}{$-0.13(-0.33$ to 0.07$)$} & \multicolumn{2}{|c|}{$-0.21(-0.43$ to 0.02$)$} & \multicolumn{2}{|c|}{$0.11(-0.11$ to 0.33$)$} & $-0.05(-$ & to 0.17 ) & $-0.14(-0$ & $00.08)$ \\
\hline AUSCAN function (0-36) & & & & & & & & & & & & \\
\hline Mean (SD) & $15.1(7.7)$ & $15.3(6.9)$ & $15.9(7.7)$ & $14.5(6.8)$ & $14.4(7.9)$ & $14.9(7.5)$ & $15.3(7.7)$ & $14.1(7.7)$ & $15.1(7.9)$ & $16.3(7.5)$ & $16.3(7.6)$ & $15.1(7.8)$ \\
\hline Adjusted mean difference $(95 \% \mathrm{Cl})$ & $-0.61(-1$ & to 0.74$)$ & $-0.70(-2$ & to 0.62 ) & $-0.36(-1$ & to 1.14$)$ & $-0.51(-2$ & to 1.02 ) & $0.51(-1$ & 2.10) & $-0.60(-2$ & o 0.98) \\
\hline AUSCAN total (0-12) & & & & & & & & & & & & \\
\hline Mean (SD) & $5.0(2.2)$ & $5.0(1.9)$ & $5.3(2.1)$ & $4.7(1.9)$ & $5.1(2.3)$ & $4.8(2.3)$ & $5.1(2.4)$ & $4.9(2.3)$ & $5.2(2.2)$ & $5.3(2.3)$ & $5.5(2.2)$ & $5.0(2.3)$ \\
\hline Adjusted mean difference $(95 \% \mathrm{Cl})$ & $-0.06(-0$ & to 0.33$)$ & $-0.25(-0$ & to 0.14$)$ & $-0.43(-0$ & to 0.06$)$ & $0.09(-0.3$ & $0.57)$ & $-0.03(-$ & to 0.46 ) & $-0.26(-0$ & $00.22)$ \\
\hline Arthritis self-efficacy for pain (1-10) & & & & & & & & & & & & \\
\hline Mean (SD) & $5.5(1.9)$ & $6.3(1.8)$ & $5.8(2.0)$ & $6.0(1.7)$ & $5.7(1.9)$ & $6.5(1.7)$ & $6.0(1.8)$ & $6.1(1.8)$ & $5.4(2.0)$ & $6.0(1.8)$ & $5.5(1.9)$ & $5.8(2.0)$ \\
\hline Adjusted mean difference $(95 \% \mathrm{Cl})$ & $0.66(0.24$ & $.08)$ & $0.16(-0.2$ & $0.58)$ & $0.74(0.30$ & 1.19) & $0.09(-0.3$ & $0.55)$ & $0.54(0.0$ & 1.02) & $0.16(-0.3$ & $0.65)$ \\
\hline Hand pain severity last 3 days $(0-10)$ & & & & & & & & & & & & \\
\hline Mean (SD) & $4.4(2.2)$ & $4.3(1.9)$ & $4.6(2.1)$ & $4.1(2.0)$ & $4.1(2.2)$ & $4.4(2.1)$ & $4.1(2.1)$ & $4.4(2.2)$ & $4.5(2.2)$ & $4.6(2.3)$ & $4.8(2.2)$ & $4.3(2.3)$ \\
\hline Adjusted mean difference $(95 \% \mathrm{Cl})$ & $-0.29(-0$ & to 0.17 ) & $-0.09(-0$ & to 0.38 ) & $0.06(-0.4$ & $0.58)$ & $0.53(0.01$ & 1.05) & $0.03(-0$ & $0.59)$ & $-0.28(-0$ & $00.27)$ \\
\hline Severity of worse problem in the last 3 & ys $(0-10)$ & & & & & & & & & & & \\
\hline Mean (SD) & $4.7(2.3)$ & $4.7(2.2)$ & $5.0(2.3)$ & $4.5(2.2)$ & $4.8(2.4)$ & $4.7(2.6)$ & $4.9(2.5)$ & $4.7(2.4)$ & $4.9(2.2)$ & $4.8(2.4)$ & $5.2(2.2)$ & $4.5(2.3)$ \\
\hline Adjusted mean difference $(95 \% \mathrm{Cl})$ & $-0.23(-0$ & to 0.30 ) & $-0.27(-0$ & to 0.24$)$ & $-0.42(-1$ & to 0.24 ) & $-0.01(-0$ & to 0.62 ) & $-0.33(-$ & to 0.23 ) & $-0.49(-1$ & $00.10)$ \\
\hline Satisfaction with hand function in the & 3 days $(0-1$ & & & & & & & & & & & \\
\hline Mean (SD) & $4.8(2.3)$ & $4.4(2.2)$ & $4.9(2.3)$ & $4.3(2.1)$ & $4.5(2.4)$ & $4.1(2.4)$ & $4.2(2.5)$ & $4.4(2.4)$ & $4.9(2.2)$ & $4.8(2.4)$ & $5.2(2.2)$ & $4.5(2.3)$ \\
\hline Adjusted mean difference $(95 \% \mathrm{Cl})$ & $-0.45(-0$ & to 0.08 ) & $-0.43(-0$ & to 0.11 ) & $-0.57(-1$ & to 0.01 ) & $0.37(-0.2)$ & $0.95)$ & $-0.19(-$ & to 0.38 ) & $-0.46(-1$ & $00.14)$ \\
\hline Grip strength (lbs) & & & & & & & & & & & & \\
\hline Mean (SD) & $\mathrm{N} / \mathrm{A}$ & N/A & $\mathrm{N} / \mathrm{A}$ & N/A & $43.2(22.8)$ & $41.0(20.8)$ & $41.3(21.7)$ & $43.0(22.0)$ & N/A & $\mathrm{N} / \mathrm{A}$ & $\mathrm{N} / \mathrm{A}$ & $\mathrm{N} / \mathrm{A}$ \\
\hline Adjusted mean difference $(95 \% \mathrm{Cl})$ & $\mathrm{N} / \mathrm{A}$ & & $\mathrm{N} / \mathrm{A}$ & & $-1.04(-4$ & to 2.07$)$ & $1.17(-2.1$ & 4.46) & $\mathrm{N} / \mathrm{A}$ & & $\mathrm{N} / \mathrm{A}$ & \\
\hline Pinch strength (lbs) & & & & & & & & & & & & \\
\hline Mean (SD) & $\mathrm{N} / \mathrm{A}$ & N/A & $\mathrm{N} / \mathrm{A}$ & N/A & $9.2(3.5)$ & $8.8(3.6)$ & $8.8(3.4)$ & $9.2(3.7)$ & N/A & $\mathrm{N} / \mathrm{A}$ & $\mathrm{N} / \mathrm{A}$ & $\mathrm{N} / \mathrm{A}$ \\
\hline Adjusted mean difference $(95 \% \mathrm{Cl})$ & $\mathrm{N} / \mathrm{A}$ & & $\mathrm{N} / \mathrm{A}$ & & $-0.27(-0$ & to 0.34$)$ & $0.33(-0.2$ & $0.95)$ & N/A & & $\mathrm{N} / \mathrm{A}$ & \\
\hline Grip ability test (seconds) & & & & & & & & & & & & \\
\hline Mean (SD) & $\mathrm{N} / \mathrm{A}$ & N/A & $\mathrm{N} / \mathrm{A}$ & $\mathrm{N} / \mathrm{A}$ & $33.9(19.2)$ & $31.3(10.8)$ & $34.5(19.4)$ & $30.8(10.6)$ & $\mathrm{N} / \mathrm{A}$ & $\mathrm{N} / \mathrm{A}$ & $\mathrm{N} / \mathrm{A}$ & $\mathrm{N} / \mathrm{A}$ \\
\hline Adjusted mean difference $(95 \% \mathrm{Cl})$ & $\mathrm{N} / \mathrm{A}$ & & $\mathrm{N} / \mathrm{A}$ & & $-1.96(-5$ & to 1.76$)$ & $-2.06(-5$ & to 1.60 ) & N/A & & $\mathrm{N} / \mathrm{A}$ & \\
\hline
\end{tabular}




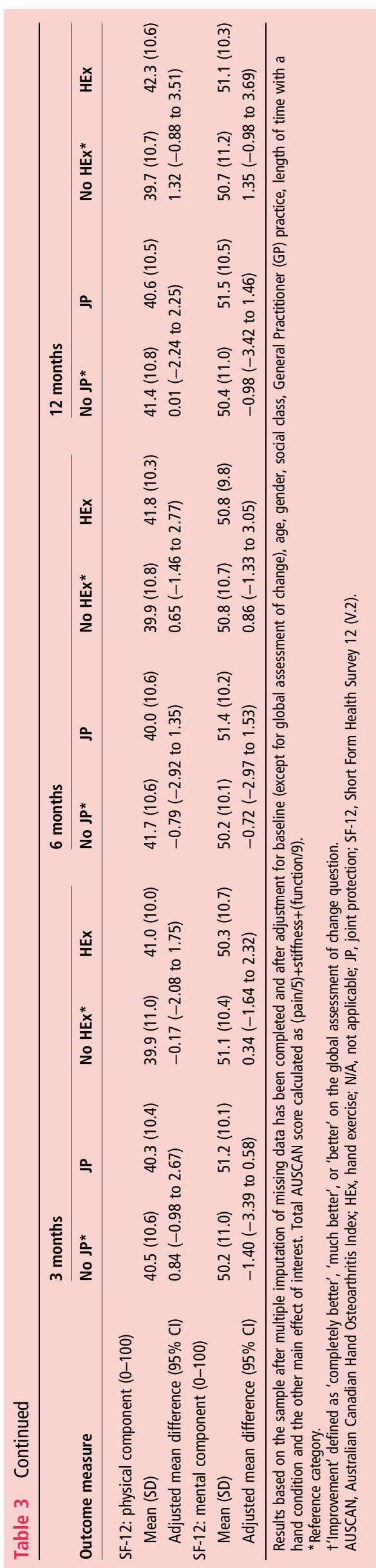

instruction in hand exercises was more effective in reducing hand pain and disability than no instruction in hand exercises, and found there was no statistically significant difference in the number of 'responders' between those receiving and not receiving hand exercises. Participants receiving joint protection education reported improved pain self-efficacy at 3-, 6- and 12months.

The research question arose from clinical practice and was shaped by OT and patient and public involvement. ${ }^{13}$ We followed the OMERACT-OARSI recommendations for design and conduct of clinical trials of OA. ${ }^{16}$ Our trial, therefore, had good internal validity with adequate sample size, high follow-up rates and strategies to minimise potential therapist effect. However, because we selected volunteers who indicated that they were able and willing to participate in an OT programme, the results may not be generalisable to all older adults with hand OA.

All participants received the same advice and leaflet from the research nurse on entry into the trial prior to randomisation, and there were no differences between groups in the timing of this. Additional intervention was delivered using the same protocol for timing of treatment appointments for each arm (no additional treatment; joint protection; hand exercises; joint protection and hand exercises) and appointments for classes were monitored. Benefit of classes may have been diluted at 3 months as some participants failed to attend the first set of classes available so were still completing classes after the 3-month follow-up.

Reviews of non-surgical treatments for hand $\mathrm{OA}^{31-33}$ and international guidelines ${ }^{7}$ have identified a gap in evidence for the effectiveness of non-pharmacological approaches in the management of hand OA in community-dwelling older adults. Guidelines acknowledge that the strength of the evidence underpinning recommendations for self-management is weak. They highlight one $\mathrm{RCT}^{12}$ comparing a joint protection programme plus home-based hand exercise (range of motion) versus information alone in 40 patients with hand OA. Stamm's study, while small, has been instrumental in informing clinical guidelines for hand OA. ${ }^{8}$ More recently, Stukstette et al ${ }^{11}$ investigated the effectiveness of an intensive group-based multidisciplinary treatment programme incorporating selfmanagement, ergonomic principles and exercises. The findings of this study in participants recruited from rheumatology clinics suggest that such a programme is not effective in the short term.

Our study has added to this evidence by investigating the independent effects of each intervention and demonstrating the effectiveness of joint protection in hand OA. All exercises were supervised in the classes by OTs experienced in treating hand OA, however, as we did not measure biomechanical outcome, we cannot determine whether our exercise programme had any effect (positive or negative) on joint deformities. Adherence to home exercises was good-those allocated to hand exercises performed a structured exercise programme more often than those who were not-but gains in grip strength and other performance measures were not shown to be statistically significant, contrary to findings of others. ${ }^{32}$ Adherence to joint protection approaches was also good, and it is possible that once joint protection principles are established, they may be easier to sustain in the shorter term, but the benefits tailor off in the longer term.

Although the magnitude of benefit for joint protection was lower than our prespecified minimally clinically important difference, it still reached statistical significance due to the additional power available in our study from an overestimation of the percentage of participants meeting the OARSI responder 
criteria in the sample size calculation. Our study, therefore, adds to the evidence for expected differences between interventions and comparators for non-pharmacological studies in community-dwelling populations, 50 years and older, with hand OA.

In clinical practice, only those patients who seek help are treated and only a small subgroup of the population with disabling hand pain in community-dwelling populations, aged 50 years and older, see an OT (3\% in a 12 -month period). ${ }^{6}$ Our eligible study population was defined using criteria recommended in secondary care settings, ${ }^{16}$ and the treatment approaches should, therefore, be generalisable to patients who are referred to OT. Our findings also highlight the feasibility of supporting self-management in community settings and offer strategies to close the gap between what patients should receive $^{7-9}$ and what therapies are offered. ${ }^{6}$

While hand $\mathrm{OA}$ is common, and has a significant impact and associated disability, ${ }^{34}$ consultations with a General Practitioner (GP) are low. ${ }^{6}$ People with hand problems consider the diagnosis of 'hand OA' to represent a serious condition, but they often perceive that nothing can be done. ${ }^{5}$ Our study population was recruited via the community using the ACR criteria for hand OA, hence, to have achieved any improvement in this group is important.

Joint pain in older adults and OA are public health problems that challenge our healthcare professionals and healthcare delivery systems. We have produced clear evidence about the most clinically effective methods of delivering and supporting self-management at 6 months for older adults with hand OA to justify and inform guidelines and recommendations. We have shown that support for self-management, through a joint protection education programme delivered by OTs, provides an effective approach for community-dwelling older adults with hand OA.

Acknowledgements The authors would like to thank Rhian Hughes and the health informatics and administrative staff at Keele University's Arthritis Research UK Primary Care Centre, especially Jo Bailey, Tracy Whitehurst, Natalie Burgess, Tracy Reynolds and Claire Calverley, staff of the participating general practices in the Keele GP Research Partnership, Bucknall Hospital (pilot study), Haywood Hospital (training), Leighton Hospital and University Hospital of North Staffordshire (main study). The authors would also like to give special thanks to all the therapists and Therapy Managers, including: Nicky Walker, Lynette Bowler, Tracey Heath, Rebecca Wood, Debbie Ferneyhough, Carol Graham, Nickie Edwards, Heather Cowley, Helen Myers, Catherine Tyson, Helen Gibbs, Louisa Whitfield, Kath Griffiths, Noeleen Hellis, Helen Duffy, Hazel Mackey and Janice Lovatt; and Ricky Mullis and David Whitehurst for their valuable input into study design and development. The authors are grateful to the members of the Research Users Group (Teresa George, Vanda Hulse, Jo Bird, Dennis Grimsley) for their patient and public involvement. Thanks to Prof. Peter Croft and Prof. Danielle van der Windt for reviewing the manuscript prior to submission. We would like to thank our anonymous reviewers for their helpful comments and suggestions. Finally, the authors would like to thank Prof. N. Bellamy for permission to use the AUSCAN.

Contributors All the authors vouch for the data and analyses as well as the fidelity of the study to the protocol. The first and second authors wrote the first draft of the manuscript; all the authors participated in writing subsequent drafts and made the decision to submit the manuscript for publication.

Funding This study was supported financially by a Project Grant awarded by Arthritis Research UK, Grant Code: 17958 and by Support for Science Funding secured by North Staffordshire Primary Care Research Consortium for NHS service support costs. The Data Monitoring Committee was conducted by Chris Roberts (Chair), Christina Jerosch Herold, and Richard McManus.

Competing interests KD was a member of the NICE OA Guideline Development Group and is a current NICE Fellow. No other potential conflict of interest relevant to this article was reported. Disclosure forms provided by the authors are available with the full text of this article at http://ard.bmj.com.

Ethics approval North West 7 Research Ethics Committee UK (rec reference: 07/H1008/235).

Provenance and peer review Not commissioned; externally peer reviewed.

Data sharing statement Our Centre has established data sharing arrangements to support joint publications and other research collaborations. Applications for access to anonymised data from our research databases are reviewed by the Centre's Data Custodian and Academic Proposal (DCAP) Committee and a decision regarding access to the data is made subject to the NRES ethical approval first provided for the study and to new analysis being proposed. Further information on our data sharing procedures can be found on the Centre's website (http://www.keele.ac.uk/ pchs/publications/datasharingresources/) or by emailing the Centre's data manager (data-sharing-pcs@cphc.keele.ac.uk).

Open Access This is an Open Access article distributed in accordance with the Creative Commons Attribution Non Commercial (CC BY-NC 3.0) license, which permits others to distribute, remix, adapt, build upon this work non-commercially, and license their derivative works on different terms, provided the original work is properly cited and the use is non-commercial. See: http://creativecommons.org/licenses/by-nc/3.0/

\section{REFERENCES}

1 Pereira D, Peleteiro B, Araujo J, et al. The effect of osteoarthritis definition on prevalence and incidence estimates: a systematic review. Osteoarthritis Cartilage 2011;19:1270-85.

2 Lawrence RC, Felson DT, Helmick CG, et al. Estimates of the prevalence of arthritis and other rheumatic conditions in the United States. Part II. Arthritis Rheum 2008; 58:26-35

3 Dillon CF, Hirsch R, Rasch EK, et al. Symptomatic hand osteoarthritis in the United States: prevalence and functional impairment estimates from the third U.S. National Health and Nutrition Examination Survey, 1991-1994. Am J Phys Med Rehabil 2007;86:12-21

4 Centers for Disease Control and Prevention. Osteoarthritis. 2011. http://www.cdc gov/arthritis/basics/osteoarthritis.htm (accessed 12 Dec 2012).

5 Hill S, Dziedzic KS, Ong BN. The functional and psychological impact of hand osteoarthritis. Chronic IIIn 2010;6:101-10.

6 Dziedzic K, Thomas E, Hill S, et al. The impact of musculoskeletal hand problems in older adults: findings from the North Staffordshire Osteoarthritis Project (NorStOP). Rheumatology (Oxford) 2007;46:963-7.

7 Zhang $W$, Doherty $M$, Leeb BF, et al. EULAR evidence based recommendations for the management of hand osteoarthritis: report of a Task Force of the EULAR Standing Committee for International Clinical Studies Including Therapeutics (ESCISIT). Ann Rheum Dis 2007;66:377-88.

8 Hochberg MC, Altman RD, April KT, et al. American College of Rheumatology 2012 recommendations for the use of nonpharmacologic and pharmacologic therapies in osteoarthritis of the hand, hip, and knee. Arthritis Care Res 2012;64:465-74

9 Conaghan PG, Dickson J, Grant RL; Guideline Development Group. Care and management of osteoarthritis in adults: summary of NICE guidance. BMJ 2008:336:502-3.

10 Pitt VJ, O'connor D, Green S. Referral of people with osteoarthritis to self-management programmes: barriers and enablers identified by general practitioners. Disabil Rehabil 2008;30:1938.

11 Stukstette MJ, Dekker J, den Broeder AA, et al. No evidence for the effectiveness of a multidisciplinary group based treatment program in patients with osteoarthritis of hands on the short term; results of a randomized controlled trial. Osteoarthritis Cartilage 2013;21:901-10.

12 Stamm TA, Machold KP, Smolen JS, et al. Joint protection and home hand exercises improve hand function in patients with hand osteoarthritis: a randomized controlled trial. Arthritis Rheum 2002;47:44-9.

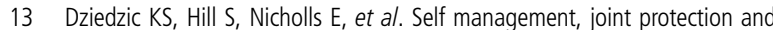
exercises in hand osteoarthritis: a randomised controlled trial with cost effectiveness analyses. BMC Musculoskelet Disord 2011;12:156.

14 Bellamy N, Campbell J, Haraoui B, et al. Dimensionality and clinical importance of pain and disability in hand osteoarthritis: Development of the Australian/Canadian (AUSCAN) Osteoarthritis Hand Index. Osteoarthritis Cartilage 2002;10:855-62.

15 Dziedzic KS, Thomas E, Myers H, et al. The Australian/Canadian osteoarthritis hand index in a community-dwelling population of older adults: reliability and validity. Arthritis Rheum 2007:57:423-8.

16 Maheu E, Altman RD, Bloch DA, et al. Design and conduct of clinical trials in patients with osteoarthritis of the hand: recommendations from a task force of the Osteoarthritis Research Society International. Osteoarthritis Cartilage 2006;14:303-22.

17 Altman R, Alarcon G, Appelrouth D, et al. The American College of Rheumatology criteria for the classification and reporting of osteoarthritis of the hand. Arthritis Rheum 1990;33:1601-10.

18 Conaghan PG, Dickson J, Grant RL, et al. Care and management of osteoarthritis in adults. BMJ 2008;336:502-3.

19 Hammond A, Freeman K. One-year outcomes of a randomized controlled trial of an educational-behavioural joint protection programme for people with rheumatoid arthritis. Rheumatology (Oxford) 2001;40:1044-51.

20 Hammond $\mathrm{A}$, Jeffreson $\mathrm{P}$, Jones $\mathrm{N}$, et al. Clinical Applicability of an Educational-Behavioural Joint Protection Programme for People with Rheumatoid Arthritis. BJOT 2002:65:405-12

21 Hammond A, Freeman K. The long-term outcomes from a randomized controlled trial of an educational-behavioural joint protection programme for people with rheumatoid arthritis. Clin Rehabil 2004;18:520-8. 


\section{Clinical and epidemiological research}

22 Hammond A, Bryan J, Hardy A. Effects of a modular behavioural arthritis education programme: a pragmatic parallel-group randomized controlled trial. Rheumatolgy (Oxford) 2008;47:1712-18.

23 van der Windt DA, Koes BW, Deville W, et al. Effectiveness of corticosteroid injections versus physiotherapy for treatment of painful stiff shoulder in primary care: randomised trial. BMJ 1998;317:1292-6.

24 Pham T, van der Heijde D, Altman RD, et al. OMERACT-OARSI initiative: Osteoarthritis Research Society International set of responder criteria for osteoarthritis clinical trials revisited. Osteoarthritis Cartilage 2004;12:389-99.

25 Ware J Jr, Kosinski M, Keller SD. A 12-Item Short-Form Health Survey: construction of scales and preliminary tests of reliability and validity. Med Care 1996:34:220-33.

26 Lorig $\mathrm{K}$, Chastain RL, Ung E, et al. Development and evaluation of a scale to measure perceived self-efficacy in people with arthritis. Arthritis Rheum 1989;32:37-44.

27 Dellhag B, Bjelle A. A Grip Ability Test for use in rheumatology practice. J Rheumatol 1995;22:1559-65.
28 Montgomery AA, Peters TJ, Little P. Design, analysis and presentation of factorial randomised controlled trials. BMC Med Res Methodol 2003;3:26.

29 Hay EM, Foster NE, Thomas E, et al. Effectiveness of community physiotherapy and enhanced pharmacy review for knee pain in people aged over 55 presenting to primary care: pragmatic randomised trial. BMJ 2006;333:995.

30 StataCorp. LP. Stata/IC 12.1 for Windows. 2012.

31 Moe RH, Kjeken I, Uhlig T, et al. There is inadequate evidence to determine the effectiveness of non-pharmacological and non-surgical interventions for hand osteoarthritis: an overview of high-quality systematic reviews. Phys Ther 2009;89:1363-70.

32 Valdes K, Marik T. A systematic review of conservative interventions for osteoarthritis of the hand. J Hand Ther 2010;23:334-50; quiz 351.

33 Kjeken I, Smedslund G, Moe RH, et al. Systematic review of design and effects of splints and exercise programs in hand osteoarthritis. Arthritis Care Res 2011;63:834-48.

34 Zhang Y, Niu J, Kelly-Hayes $M$, et al. Prevalence of symptomatic hand osteoarthritis and its impact on functional status among the elderly: the Framingham Study. Am J Epidemiol 2002;156:1021-7. 